Document downloaded from:

http://hdl.handle.net/10251/105465

This paper must be cited as:

Vila Tortosa, MP.; Baeza González, LM.; Martínez Casas, J.; Carballeira, J. (2014). Rail corrugation growth accounting for the flexibility and rotation of the wheel set and the nonHertzian and non-steady-state effects at contact patch. Vehicle System Dynamics. 52:92108. doi:10.1080/00423114.2014.881513

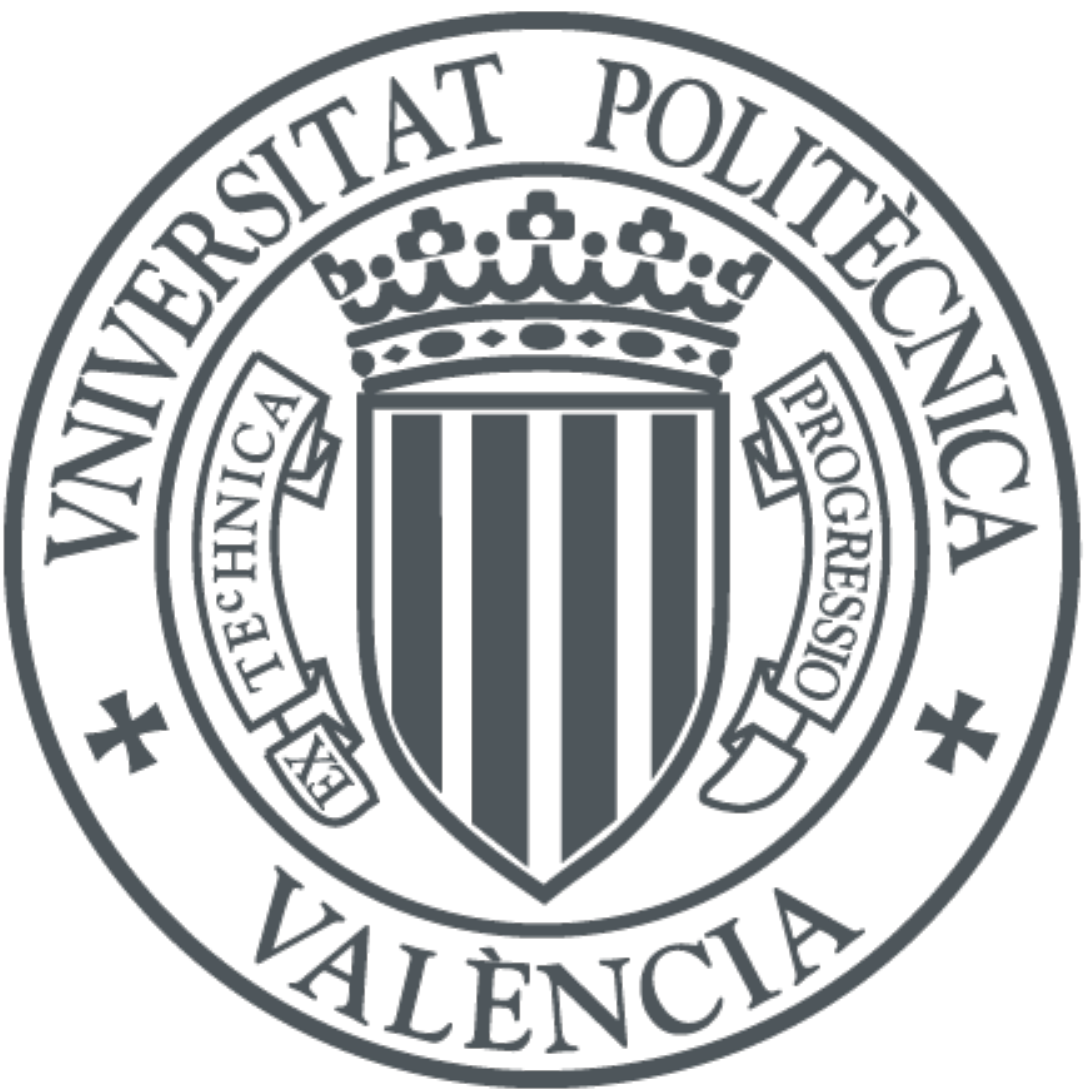

The final publication is available at

https://doi.org/10.1080/00423114.2014.881513

Copyright Taylor \& Francis

Additional Information 


\title{
Rail corrugation growth accounting for the flexibility and rotation of the railway wheelset and the non-Hertzian and non-steady-state effects at contact patch.
}

\author{
Paloma Vila, Luis Baeza*, José Martínez-Casas and Javier Carballeira \\ Centro de Investigación de Tecnología de Vehículos, Universitat Politècnica de València \\ Camino de Vera $\mathrm{s} / \mathrm{n}$, \\ 46022 Valencia, Spain \\ *baeza@mcm.upv.es
}

\begin{abstract}
In this work, a simulation tool is developed to analyse the growth of rail corrugation consisting of several models connected in a feedback loop in order to account for both the short-term dynamic vehicle-track interaction and the long-term damage. The time-domain vehicle-track interaction model comprises a flexible rotating wheel set model, a cyclic track model based on a substructuring technique and a non-Hertzian and non-steady-state three-dimensional wheel-rail contact model, based on the variational theory by Kalker.Wear calculation is performed with Archard's wear model by using the contact parameters obtained with the non-Hertzian and non-steady-state three-dimensional contact model. The aim of this paper is to analyse the influence of the excitation of two coinciding resonances of the flexible rotating wheel set on the rail corrugation growth in the frequency range from 20 to $1500 \mathrm{~Hz}$, when contact conditions similar to those that can arise while a wheelset is negotiating a gentle curve are simulated. Numerical results show that rail corrugation grows only on the low rail for two cases in which two different modes of the rotating wheel set coincide in frequency. In the first case, identified by using the Campbell diagram, the excitation of both the backward wheel mode and the forward third bending mode of the wheel set model (B-F modes) promotes the growth of rail corrugation with a wavelength of $110 \mathrm{~mm}$ for a vehicle velocity of $142 \mathrm{~km} / \mathrm{h}$. In the second case, the excitation of both the backward wheel mode and the backward third bending mode (B-B modes) gives rise to rail corrugation growth at a wavelength of $156 \mathrm{~mm}$ when the vehicle velocity is $198 \mathrm{~km} / \mathrm{h}$.
\end{abstract}

Keywords: rail corrugation; flexible rotating wheel set; cyclic track; global corrugation growth rate

\section{INTRODUCTION}

Rail corrugation remains nowadays, after a long time of research and field observations, one of the most severe problems faced by railway administrations around the world. The reason for this can lie in the wide diversity of rail corrugation, which is probably produced by different causes. The most accepted classification of rail corrugation is due to Grassie and Kalousek, who classified various types of rail corrugation with respect to wavelength-fixing mechanisms and damage mechanisms [1].

Several authors have concluded in their investigations that the formation and development of rail corrugation can be attributed to certain resonances and antiresonances of the coupled vehicle-track system, which act as wavelength-fixing mechanisms. Some examples of these wavelength-fixing mechanisms identified so far are the pinned-pinned vertical resonance of the track (and the corresponding antiresonance above the sleepers) [2-4]; the P2 resonance of the track, in which the rails and sleepers vibrate in phase on the ballast [4,5]; the vertical antiresonance of the track in which the sleepers vibrate independently as dynamic vibration absorbers [3,5]; the first and second torsional modes of the wheelset axle [4]; and the bending modes of the stretch of rail between the wheelsets of a bogie [6]. In some cases, the coincidence of two or more resonances or antiresonances of the vehicle-track system in the same frequency range can have a synergistic effect that can magnify the growth of rail corrugation at certain wavelengths. Just to mention some of them: the frequency match of the first vertical 
antiresonance and the first pinned-pinned lateral resonance of the track [7]; the coincidence in the same frequency interval of the P2 resonance and the first vertical antiresonance of the track [8]; and the combination of a vertical antiresonance and a lateral resonance of the track with a resonance of the wheelset [9]. Frequently, wear is the only damage mechanism taken into account, since it is known to be responsible for most types of rail corrugation [1].

In this context, simulation tools of rail corrugation can play a key role in gaining a better understanding of the initiation and growth of this defect. In this work, the simulation tool developed to analyse the growth of rail corrugation consists of several models related to each other by means of a feedback loop to account both for the short-term dynamic vehicle-track interaction, and for the long-term damage. The vehicle-track interaction model comprises a flexible rotating wheelset model [10], a cyclic track model based on a substructuring technique [11] and a non-Hertzian and non-steady state three-dimensional wheel-rail contact model, based on the variational theory by Kalker [12]. The dynamic interaction between the vehicle and the track is solved in the time domain to take into account the nonlinearities of the coupled vehicle-track system. Wear calculation is performed in a post-process stage, using the non-Hertzian and non-steady three-dimensional contact model, which is able to represent adequately the distributions of contact stress and slip velocity, which are required as input data to the Archard's wear model to compute the material loss on the running surfaces of the two rails.

The use of a non-Hertzian and non-steady state contact model to calculate wear has been proved by several authors [13-15] to lead to no growth of rail corrugation, since the maximum wear depths are located near the corrugation peaks. This fact may be caused by the geometrical shift of the contact point when the wheel rolls over a corrugated rail, which has been demonstrated to move the wear maxima towards the peaks of the corrugation [16]. However, these results are completely opposite when compared to those obtained with Hertz/FASTSIM models, which predict corrugation growth in most cases. The causes of these differences can be attributed to the simplifying assumptions made in Hertz/FASTSIM models, which cannot take into account the real shape of the contact patch, asymmetrical stress distributions in contact patch, geometric shift of the contact patch and the non-steady effects produced by the high frequency fluctuations of the contact parameters. These shortcomings are overcome in this work by implementing a non-Hertzian and non-steady state contact model.

Continuing previous work in [17], this paper analyses the influence of the excitation of two coinciding resonances of a flexible rotating wheelset on the corrugation growth in the frequency range from $20 \mathrm{~Hz}$ to $1500 \mathrm{~Hz}$. It must be highlighted that there are few papers in the literature concerning rail corrugation that implement the flexibility and the gyroscopic effects associated with the rotation of a railway wheelset since, typically, they were modelled as rigid masses or only the flexibility of the wheelset axle is considered. In [7] a flexible wheelset was implemented but the rotation was neglected and in [15] the former features were accounted for but only the wheelset axle was model as flexible and rotating since the wheels were represented as rigid bodies.

Contact conditions similar to those that can arise when a wheelset is negotiating a high radius curve are prescribed to analyse the potential growth of rail corrugation. To this aim, two different excitation irregularities on the rail running surfaces are accounted for: sinusoidal rail corrugation and broadband rail roughness. 


\section{VEHICLE-TRACK INTERACTION}

In this work, the time-domain vehicle-track interaction model consists of the cyclic track model described in [11] and the flexible and rotating wheelset model developed in [10]. The forces transmitted through the wheel-rail contact are calculated by means of a three-dimensional non-Hertzian and non-steady contact model based on the Kalker's variational theory [12], which is implemented into the time- stepping algorithm.

The modelling of the dynamic vehicle-track interaction is based on a substructuring technique, which considers three different types of substructures: vehicle, rails and sleepers. Each substructure is defined by a set of ordinary differential equations. The connection among the different substructures is done through the external forces acting on each substructure. These external forces are both the wheel-rail contact forces, which are applied on the vehicle and the rails, and the railpad forces, acting on the rails and sleepers.

\subsection{Track model}

The cyclic track model used in this research work can be interpreted as an infinite track on which an infinite number of identical vehicles circulate at the same velocity $V$. Thus, thanks to the periodicity properties, the analysis of an infinite track is reduced to the study of a stretch of track with a finite length $L$, which is the distance between two consecutive vehicles, as shown in Figure 1. The track length considered is $36 \mathrm{~m}$, corresponding to 60 sleeper bays.
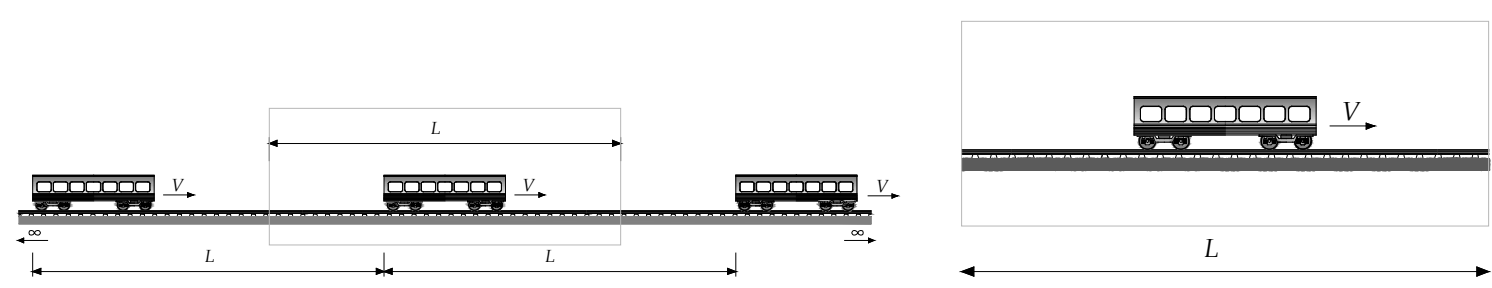

Figure 1. Cyclic track model

Rails are modelled as cyclic Timoshenko beams with the properties of the UIC60 rail and their mode shapes are calculated analytically through harmonic functions [11]. To represent the vibration of the rail, 60 vertical vibration modes are included, with frequencies up to $2.5 \mathrm{kHz}$. Sleepers are considered as rigid bodies. Railpads and ballast are modelled as viscous-elastic elements, whose stiffness and damping in the vertical direction along with other track parameters are summarised in Table 1. In Figure 2 the substructures of the track model are represented together with the connection elements. 

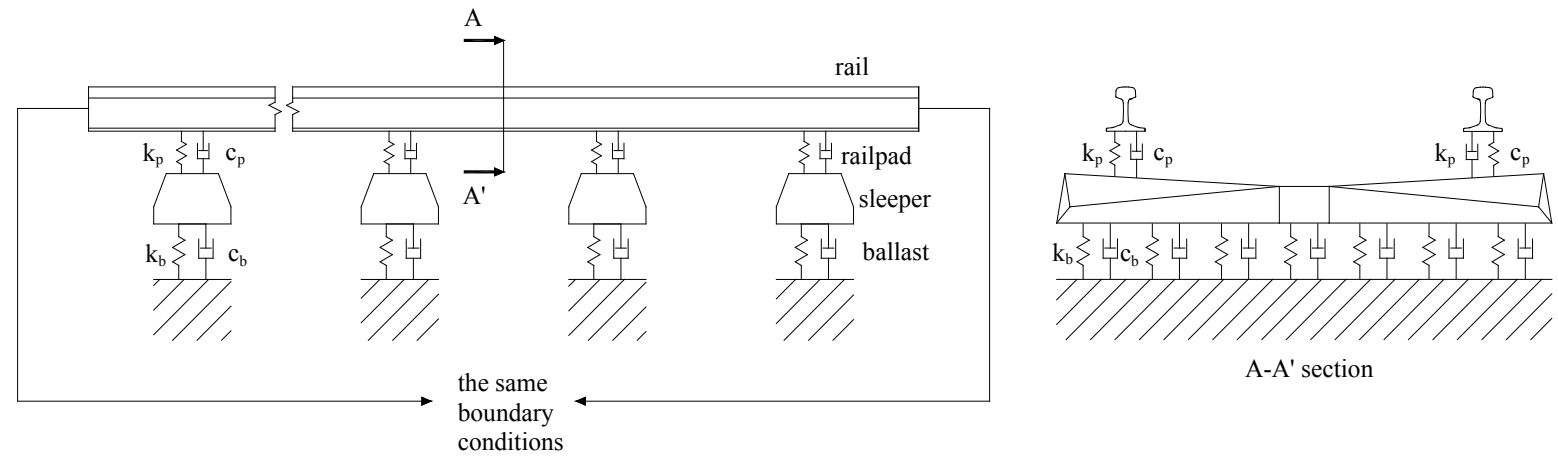

Figure 2. Track model consisting of the substructures rails and sleepers connected by visco-elastic elements that represent the railpads and the ballast.

Table 1.Parameters of the cyclic track model.

\begin{tabular}{lc}
\hline Track parameters & Value \\
\hline Rail & 60.34 \\
Mass $(\mathrm{kg} / \mathrm{m})$ & $7.68710^{-3}$ \\
Cross-section area $\left(\mathrm{m}^{2}\right)$ & 210 \\
Young's modulus $(\mathrm{GPa})$ & 81 \\
Shear modulus $(\mathrm{GPa})$ & 1.077 \\
Bending stiffness about vertical axis $\left(\mathrm{MNm}^{2}\right)$ & 6.416 \\
Bending stiffness about lateral axis $\left(\mathrm{MNm}^{2}\right)$ & 0.16 \\
Saint-Venant torsional stiffness $\left(\mathrm{MNm}^{2}\right)$ & 0.4 \\
Timoshenko shear coefficient $(\mathrm{vertical} \mathrm{and} \mathrm{lateral)}$ & \\
Railpad & 300 \\
$\quad$ Vertical stiffness, $\mathrm{k}_{\mathrm{p}}(\mathrm{MN} / \mathrm{m})$ & 30 \\
Damping coefficient, $\mathrm{c}_{\mathrm{p}}(\mathrm{kNs} / \mathrm{m})$ & \\
Sleeper & 324 \\
Mass $(\mathrm{kg})$ & 0.6 \\
Sleeper spacing $(\mathrm{m})$ & \\
Ballast & 160 \\
Vertical stiffness, $\mathrm{k}_{\mathrm{b}}(\mathrm{MN} / \mathrm{m})$ & 200 \\
Damping coefficient, $\mathrm{c}_{\mathrm{b}}(\mathrm{kNs} / \mathrm{m})$ & \\
\hline
\end{tabular}

The set of ordinary differential equation in modal coordinates related to the sth substructure can be expressed as follows

$$
\ddot{q}_{n}^{s}(t)+2 \xi_{n}^{s} \omega_{n}^{s} \dot{q}_{n}^{s}(t)+\left(\omega_{n}^{s}\right)^{2} q_{n}^{s}(t)=f_{n}^{s}(t),
$$


in which $q_{n}^{s}$ is the modal coordinate, $\omega_{n}^{s}$ is the natural frequency, $\xi_{n}^{s}$ is the spectral damping and $f_{n}^{s}$ is the modal force associated with the $n$th vibration mode of the model. More details on the track model can be found in $[11,17]$.

\subsection{Wheelset model}

When the dynamic interaction is analysed in the high frequency range, assuming that the suspensions isolate effectively the wheelset from the car body at frequencies higher than $20 \mathrm{~Hz}$, the model of the vehicle can be reduced to the model of the wheelset (unsprung masses) with the proportional part of the car body weight. Popp et al. [18] highlighted the need for considering the wheelset flexibility and gyroscopic effects related to rotation in models of vehicle-track interaction in the medium- and high-frequency ranges, since the elastic deformations of the wheelset may affect the calculation of the contact creepages, and consequently the determination of wear, and the gyroscopic effects are responsible for the splitting of the wheelset resonance peaks associated with mode shapes with multiplicity two.

The wheelset model used in this work is based on a general method for rotating solids of revolution presented in [10] and considers the flexibility and the gyroscopic effects due to rotation. The fundamental idea of this method is that the deformed shape of the solid of revolution can be defined through the modal functions in non-rotating coordinates, thanks to the properties of solids of revolution. Thus, the relationship between the displacements due to the deformation $\mathbf{u}$ and the modal coordinates $\mathbf{q}_{w}$ is given by the modal function matrix $\Phi$ through

$$
\mathbf{u}=\Phi \mathbf{q}_{w} .
$$

The differential equation governing the movement of the wheelset is

$$
\ddot{\mathbf{q}}_{w}-2 \Omega \widetilde{\mathbf{G}} \dot{\mathbf{q}}_{w}+\left(\widetilde{\mathbf{K}}+\Omega^{2} \widetilde{\mathbf{C}}\right) \mathbf{q}_{w}=\mathbf{Q}_{q}+\Omega^{2} \widetilde{\mathbf{L}},
$$

where $\Omega$ is the rotating velocity of the wheelset, $\widetilde{\mathbf{G}}$ is the gyroscopic matrix, $\widetilde{\mathbf{K}}$ is a diagonal matrix containing the square of the natural frequencies of the non-rotating wheelset, $\widetilde{\mathbf{C}}$ is a matrix which implements the centripetal forces that depend on the deformation of the solid and $\tilde{\mathbf{L}}$ takes account of the centripetal forces associated with the undeformed shape. The generalised force vector $\mathbf{Q}_{q}$ is calculated from the external forces through the matrix of modal functions. All these matrices are constant and are calculated once at the beginning of the simulation of the vehicle-track interaction. For further details on the formulation of the wheelset model see [17]. Table 2 contains the values of the parameters of the wheelset model.

Table 2. Parameters of the flexible rotating wheelset model

\begin{tabular}{lc}
\hline Wheelset parameters & Value \\
\hline Wheelset mass $(\mathrm{kg})$ & 2700 \\
Static load $(\mathrm{kN})$ & 200 \\
Rolling radius of the wheel $(\mathrm{m})$ & 0.5 \\
Moment in the vertical and lateral axes $\left(\mathrm{kg} \mathrm{m}^{2}\right)$ & 493 \\
Moment in the rolling axis $\left(\mathrm{kg} \mathrm{m}^{2}\right)$ & 169 \\
\hline
\end{tabular}


In the Campbell diagram, the equivalent resonance frequencies of the mounted wheelset are represented in function of the velocity of the vehicle. The model of the flexible rotating wheelset used in this research work considers the gyroscopic effects due to wheel rotation, which cause a resonance peak of a mode shape of multiplicity two to split into two different peaks associated with the backward and forward whirl modes, with frequencies lower and higher, respectively, than the resonance frequency corresponding to zero velocity. It has been identified two cases in which two wheelset resonance frequencies coincide. They result from the intersection of the line of the backward wheel mode with two nodal diameters and no nodal circle with the lines corresponding to the forward (B-F modes) and backward third bending modes (B-B modes), as can be seen in Figure 3. These mode shapes are shown in Table 3.

In the following, the effects of the excitation of these two coinciding resonances of the flexible rotating wheelset on the corrugation growth will be analysed.

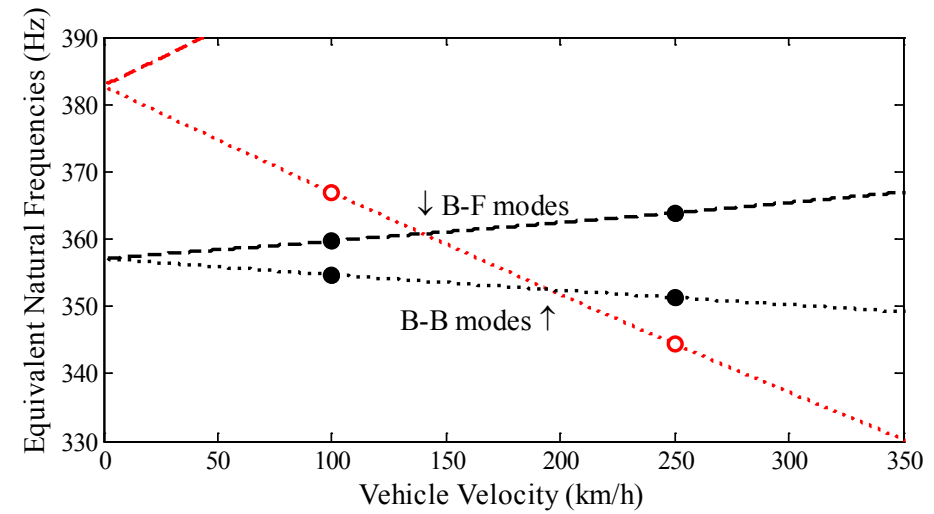

Figure 3. Zoom of the Campbell diagram for the railway wheelset [17]: o wheel modes (---:forward wheel modes, '..... backward wheel modes); - third bending modes (---·forward third bending mode, ...... backward third bending mode).

\subsection{Wheel-rail contact model}

The non-Hertzian and non-steady three-dimensional wheel-rail contact model used in this work is based on the variational theory by Kalker [12]. The main disadvantage in using this contact model is its high computational cost, that makes it difficult to obtain the results by discretisating the potential contact area in a very refined mesh, and consequently, by adopting a small time step in the time-stepping integration algorithm.

The discretisation of the potential contact area, which is considered in this paper with hexagonal shape, is done with piecewise linear triangular elements following a suggestion made by Johnson in order to improve the representation of the contact traction distributions [19]. In Figure 4, the Johnson's idea is illustrated for the bidimensional case. By changing from non-overlapping rectangular elements, in which the tractions are assumed constant, to overlapping triangular elements, in which the tractions vary linearly, it is pursued an enhanced representation of the distributions of tractions in the contact patch. 


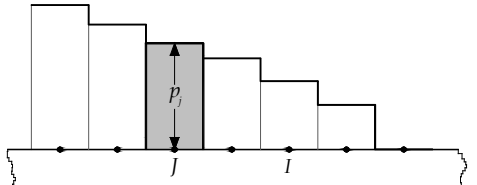

(a)

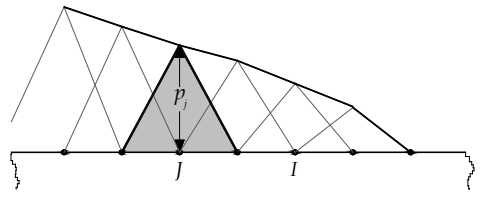

(b)

Figure 4. Approximation of the surface traction through: (a) Piecewise constant pressure elements; (b) piecewise linear overlapping pressure elements.

In the three-dimensional contact problem this idea is equivalent to obtaining the distributions of tractions in the contact patch through the superposition of overlapping pyramidal pressure elements on hexagonal bases formed by six equilateral triangular elements [19], so that in each triangular element the tractions vary linearly, as shown in Figure 5.

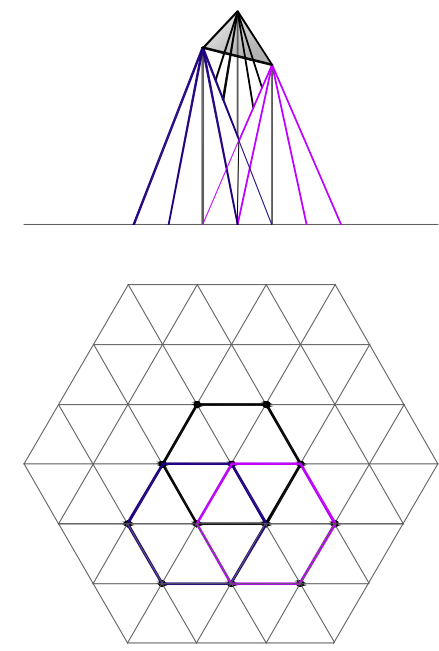

Figure 5. Approximation of the distributions of tractions through the superposition of overlapping pyramidal pressure elements on hexagonal bases formed by six equilateral triangles.

A cartesian coordinate system $\left(x_{1} x_{2} x_{3}\right)$ with the origin at the centre of the wheel-rail contact is assumed, with the $x_{1}$-axis pointing in the longitudinal direction of the track, the $x_{2}$-axis pointing in the lateral direction and the $x_{3}$-axis pointing vertically upwards. The following development is based on the assumption that both the wheel and the rail behave as elastic half-spaces. This assumption is only valid for the contact between the rail crown and the wheel tread, which takes place when vehicles run on straight tracks or gentle curves.

In this paper, the elastic influence coefficients are calculated at the vertices of the triangular elements (nodes) by integrating the Boussinesq-Cerruti constitutive equations over triangular domains loaded with linearly varying distributions of tractions, which vary from unity at one vertex to zero at the other two vertices of the triangle. An elastic influence coefficient can be denoted as $D_{I i J j}$ and it enables obtaining the elastic displacement in the $i$-direction at the node $I$, which is produced by a load applied in the $j$-direction at the node $J$.

For a pyramidal load of unitary value acting at node $J$ in the $j$-direction, the elastic influence coefficients are obtained at the mesh nodes (vertices of the triangles) by adding the contributions of the 6 triangles which form the hexagonal base of the pyramid 


$$
D_{I i J j}=\sum_{T=1}^{6} D_{I i J j}^{T}, \quad i, j=1,2,3
$$

Thus, the contact parameters, such as contact tractions, local slip velocities and displacements, are obtained at the $N$ inner nodes of the potential contact area. Considering the quasi-identity properties of the materials of wheel and rail, the normal and the tangential contact problem can be solved independently. First, the normal contact problem is solved to determine the real contact patch and the distribution of normal contact pressure. The normal contact force is calculated by integrating the distribution of normal contact pressure over the contact patch. Next, once the contact patch is known, the tangential contact problem is solved. Solving the tangential problem consist in determining which nodes are either in the stick area and which ones are in the slip area and obtaining the distribution of tangential contact tractions. The longitudinal and lateral contact forces are calculated through the integration of the longitudinal and lateral distributions of contact tractions, respectively.

Table 3. Third bending modes and wheel modes of the wheelset

\begin{tabular}{llll}
\hline \multicolumn{1}{c}{ Modes } & $\begin{array}{c}\text { Frequency }(\mathrm{Hz}) \\
(\mathrm{V}=0 \mathrm{~km} / \mathrm{h})\end{array}$ & Multiplicity & Mode shape \\
\hline $\begin{array}{l}\text { Third horizontal } \\
\text { and vertical } \\
\text { bending modes }\end{array}$ & 357 & \\
\hline $\begin{array}{l}\text { Modes of the } \\
\text { wheel with two } \\
\text { nodal diameters } \\
\text { and no nodal } \\
\text { circle }\end{array}$
\end{tabular}

\subsubsection{Formulation of the normal contact problem}

The deformed distance between wheel and rail at a node $I$ of the potential contact area is defined as the difference of displacements between these bodies in the normal direction $u_{I 3}$ plus the undeformed distance between them $h_{I}$, which accounts for the irregularities existing in the running surfaces of rails

$$
e_{I}=u_{I 3}+h_{I},
$$

where the difference of displacements $u_{I 3}$ can be obtained through the elastic coefficients $D_{I 3 J 3}$ and the normal pressure acting on the $N$ nodes of the potential contact area as 


$$
u_{I 3}=\sum_{J=1}^{N} D_{I 3 J 3} p_{J 3}
$$

Thus, the normal contact problem can be solved using an iterative process, by defining an active set consisting of the nodes that are outside the real contact area and an inactive set formed by the nodes belonging to the contact area. From the conditions of the minimisation problem of the complementary energy, the normal contact problem can be formulated in function of the Lagrangian multipliers $\lambda_{I 3}$, which coincide with the deformed distance for the normal contact problem, as

$$
\begin{aligned}
& e_{I}=\lambda_{I 3}=\sum_{J=1}^{N} D_{I 3 J 3} p_{J 3}+h_{I} \\
& \text { In contact area: } p_{I 3}>0 \text { y } \lambda_{I 3}=e_{I}=0 \quad \text { (inactive set) } \\
& \text { Outside contact area: } p_{I 3}=0 \text { y } \lambda_{I 3}=e_{I}>0 \quad \text { (active set) }
\end{aligned}
$$

The solution for the normal contact problem is obtained when all the nodes of the potential contact area satisfy the conditions given in Equation (7).

\subsubsection{Formulation of the tangential contact problem}

The kinematic equation for the tangential contact problem relates the local shift $S_{\tau}$, which is the relative displacement between the wheel and rail associated to a time increment $\Delta t$, with the local rigid shift $W_{\tau}$, the tangential displacements in the contact patch due to the current distribution of the tangential tractions $u_{I \tau}$ and the displacements produced in the contact patch by the tangential tractions in a previous time step $u_{I \tau}^{\prime}$

$$
S_{I \tau}=W_{I \tau}+u_{I \tau}-u_{I \tau}^{\prime}, \quad \tau=1,2,
$$

where the local rigid shift $W_{\tau}$ is obtained from the longitudinal $\xi$, lateral $\eta$ and spin $\phi$ creepages, the coordinates of the node $I$ with respect to the centre of the potential contact area and the spatial step $\Delta x=V \Delta t$, which is the distance that the potential contact area is moved forward in each time step

$$
W_{I 1}=\Delta x\left(\xi-y_{I} \phi\right) ; \quad W_{I 2}=\Delta x\left(\eta+x_{I} \phi\right)
$$

The differences of tangential displacements $u_{I \tau}$ are calculated by means of the elastic influence coefficients $D_{I \pi / K}$ and the tangential tractions applied on each of the $N$ nodes of the potential contact area through

$$
u_{I \tau}=\sum_{\kappa=1}^{2} \sum_{J=1}^{N} D_{I \tau / \kappa} p_{J \kappa}, \tau=1,2
$$

The tangential contact problem is solved by means of an iteration process, which takes into account an active set, constituted by the nodes in the stick area, and an inactive set, consisting of the nodes in the slip area. The tangential contact problem can be formulated in function of the Lagrangian multipliers $\lambda_{I \tau}$, considering the conditions of the minimisation problem of the complementary energy, as 


$$
\begin{aligned}
& S_{I \tau}=\sum_{\kappa=1}^{2} \sum_{J=1}^{N} D_{I \tau \sigma} p_{J \kappa}+W_{I \tau}-u_{I \tau}^{\prime}=-\lambda_{I \tau} \frac{p_{I \tau}}{\left|\mathbf{p}_{I \tau}\right|}, \quad \tau=1,2 \\
& \text { In stick area: }\left|\mathbf{p}_{I \tau}\right|<\mu p_{I 3} \text { y } \lambda_{I \tau}=\left|\mathbf{S}_{I \tau}\right|=0 \quad \text { (active set) } \\
& \text { In slip area: }\left|\mathbf{p}_{I \tau}\right|=\mu p_{I 3} \text { y } \lambda_{I \tau}=\left|\mathbf{S}_{I \tau}\right|>0 \quad \text { (inactive set) }
\end{aligned}
$$

After each iteration, the active set is updated and the solution is reached when all nodes in the contact area fulfill the conditions established in Equation (11). The friction coefficient is assumed constant and equal to 0.35 .

Finally, the contact forces are calculated as the superposition of the volumes of pyramids of height $p_{I \kappa}$ with hexagonal base, which is formed by six equilateral triangles with sides of length $l_{t}$

$$
F_{\kappa}=\frac{1}{3} A_{\text {hex }} \sum_{I=1}^{N} p_{I \kappa}=\frac{\sqrt{3}}{2} l_{t}^{2} \sum_{I=1}^{N} p_{I \kappa}, \kappa=1,2,3
$$

\subsection{Modelling of the initial rail irregularities}

In this work, two kinds of irregularities on the running surfaces of rails are implemented for modelling the excitation of the vehicle-track system: sinusoidal corrugations and broadband roughness, as illustrated in Figure 6.

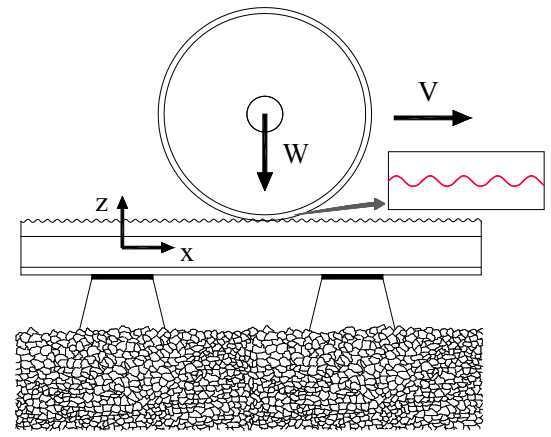

(a)

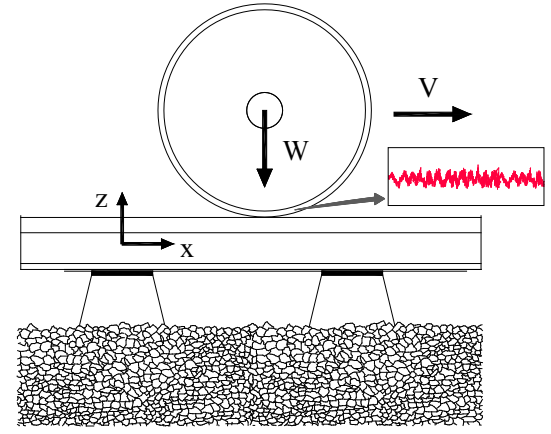

(b)

Figure 6. Excitation of the vehicle-track system through: (a) Sinusoidal corrugation; (b) Broadband rail roughness.

\subsubsection{Sinusoidal rail corrugations}

In order to excite the B-B and the B-F wheelset modes, initial sinusoidal corrugations are assumed to be present on the running surfaces of rails with wavelengths calculated as the velocity divided by the frequency corresponding to the crossing points on the Campbell diagram. Table 4 presents the frequencies of the B-F and B-B modes together with the wavelengths and amplitudes of the rail corrugations used to excite them and the vehicle velocities. 
Table 4. Frequencies and vehicle velocities of the studied modes and characteristics of the rail corrugation used to excite them.

\begin{tabular}{lcccc}
\hline & $\begin{array}{c}\text { Frequency } \\
(\mathrm{Hz})\end{array}$ & $\begin{array}{c}\text { Velocity } \\
(\mathrm{km} / \mathrm{h})\end{array}$ & $\begin{array}{c}\text { Corrugation } \\
\text { wavelength }(\mathrm{mm})\end{array}$ & $\begin{array}{c}\text { Corrugation } \\
\text { amplitude }(\mu \mathrm{m})\end{array}$ \\
\hline B-F modes & 360.8 & 142 & 110 & 10 \\
B-B modes & 352.5 & 198 & 156 & 10 \\
\hline
\end{tabular}

\subsubsection{Broadband rail roughness}

In this paper, the limit roughness spectrum on the running surfaces of rails established in the standard ISO 3095:2005 [20] is implemented to reproduce a realistically low level of roughness containing a broadband spectrum of wavelengths, ranging from $0.00315 \mathrm{~m}$ to $0.63 \mathrm{~m}$. This standard indicates the roughness level $L_{r i}$, in decibels, for the central wavelengths $\lambda_{i}$ of twenty-four $1 / 3$ octave bands

$$
\lambda_{i}=0.01 \cdot 10^{k / 10}, \text { with } k=-5,-4, \ldots, 18 \text { and } i=1, \ldots, 24
$$

The initial roughness profile can be calculated, following the procedure used by Hiensch et al. [21], as the superposition of sinusoidal functions whose amplitudes are calculated for each $1 / 3$ octave band from the levels of the rail roughness spectrum

$$
z_{0}(x)=\sum_{i=1}^{M} a_{i}\left[\sum_{j=1}^{N} \sin \left(\frac{2 \pi x}{\lambda_{i j}}+\varphi_{i j}\right)\right],
$$

where $M$ is the number of $1 / 3$ octave bands and $N$ is the number of sinus functions used to obtain the roughness profile. The $M$ amplitudes $a_{i}$, in $\mu \mathrm{m}$, associated with each band are calculated in the function of the roughness level corresponding to the band

$$
a_{i}=\sqrt{\frac{2}{N}} 10^{L_{r i} / 20}
$$

The phase angles $\varphi_{i j}$ are obtained as random numbers uniformly distributed from 0 to $2 \pi$. The $N$ wavelengths in each band $\lambda_{i j}$ are calculated by taking into account a constant increment of the wave number

$$
\Delta \kappa_{i}=\frac{2 \pi}{N}\left(\frac{1}{\lambda_{i}^{\min }}-\frac{1}{\lambda_{i}^{\max }}\right),
$$

with $\lambda_{i}^{\min }$ and $\lambda_{i}^{\max }$ being the wavelengths of the extremes of each band, which are calculated through the wavelength of the centre of each band $\lambda_{i}$ as follows

$$
\lambda_{i}^{\max }=\lambda_{i} 2^{1 / 6} ; \lambda_{i}^{\min }=\frac{\lambda_{i}}{2^{1 / 6}}
$$

In this work, twenty-four $1 / 3$ octave bands and 100 sinus functions have been considered to represent the initial rail roughness. 


\section{SIMULATION OF RAIL WEAR}

\subsection{Wear model}

Archard's wear model states that the volume of material which is removed from a surface due to wear is directly proportional to the normal load and the sliding distance between the bodies in contact. From the model of Archard, an expression for estimating the wear depth at a node $I$ of the contact patch can be deduced

$$
\Delta z_{I}=\frac{k_{\mathrm{w}}}{H} p_{I 3}\left|S_{I}\right|
$$

in which $p_{I 3}$ is the normal contact pressure at node $I$ and $\left|S_{I}\right|$ is the norm of the local shift, which is the sliding distance obtained by multiplying the slip velocity and the time step, $H$ is the hardness of the softer material and $k_{\mathrm{w}}$ is the wear coefficient. In this paper, the wear coefficient is assumed independent of the variations of the normal contact pressure and the sliding velocity in the contact patch. A wear coefficient of $1.09 \cdot 10^{-4}$, which corresponds to low sliding velocities, and a hardness of $3.2 \mathrm{GPa}$ have been used for the simulations of railhead wear.

\subsection{Rail profile updating}

In order to map the wear depth distributions $\Delta z$ in the contact area onto the rail, the running surfaces of the rails are discretised into a mesh of nodes uniformly distributed in the longitudinal and lateral direction. Wear depths in the rail nodes are calculated by displacing the potential contact area a distance $\Delta x=V \Delta t$, which is equal to the traversed distance in each time step, at the vehicle velocity $V$. If, for a certain time instant $i$, a node of the railhead mesh $k$ is located within the contact area, the wear depth in such rail node is calculated by means of a linear interpolation of the values of wear depth at the three vertices of the triangle containing the rail node. For each time step, the wear depths in the rail nodes are saved. Thus, the total wear depth in a rail node is calculated by addition of the wear depth for each time step for all the time steps $N_{t}$ considered, as follows

$$
\Delta z^{k}=\sum_{i=1}^{N_{t}} \Delta z_{i}^{k}
$$

Then, the updated rail profile after $n$ wheelset passages is obtained by subtracting the wear depth for a wheelset passage from the initial (or previous) rail profile

$$
z_{n}=z_{n-1}-\Delta z
$$

\section{NUMERICAL RESULTS}

\subsection{Initial sinusoidal corrugation}

In the simulation tool, it has been implemented contact conditions similar to those that can be found when a vehicle passes through a gentle curve with a high radius, in which the contact predominantly takes place between the rail crown and the wheel tread and flange contact rarely, if ever, occurs. In order to simulate the forces arising in the wheel-rail contact due to curve negotiation, mean values for the longitudinal creepages have been prescribed. It has been 
considered a positive mean value of $0.2 \%$ for modelling the contact conditions between the inner wheel and the low rail, while a negative mean value of $-0.2 \%$ has been adopted to represent the conditions in the contact between the outer wheel and the high rail. According to some works in the literature [22] these values can be considered reasonable as an initial approximation to the problem addressed in this study for the speeds of the vehicle taken into account. These contact conditions are represented in Figure 7.

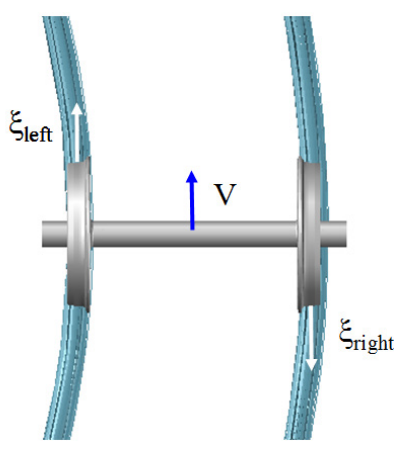

Figure 7. Contact conditions adopted to simulate the passing of the wheelset through a high radius curve

Figure 8 shows the wear depths on the centre line of the running surface of both rails of the curved track when the B-F modes are excited with a sinusoidal rail corrugation with wavelength $110 \mathrm{~mm}$ and amplitude $10 \mu \mathrm{m}$. The wear depths on both rails are compared with the initial rail corrugation, which is represented out of scale for the sake of clarity. The phase angle between the wear depth obtained from the simulation tool and the initial rail corrugation is an important parameter that is usually taken into account in order to analyse the potential growth of rail corrugation $[13,15]$. Thus, as can be observed in Figure 8(a), corresponding to the low rail, the wear depth is approximately in anti-phase with respect to the initial corrugation, which indicates that rail corrugation could grow on the low rail under the studied conditions. However, as can be deduced from Figure 8(b), no rail corrugation growth is predicted for the high rail, since the wear depth is almost in-phase with the initial rail corrugation, which means that the corrugation crests would be removed after thousands of wheel passages.

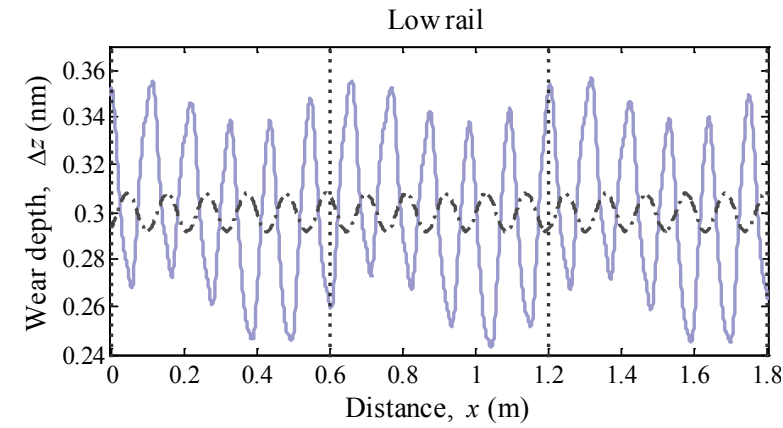

(a)

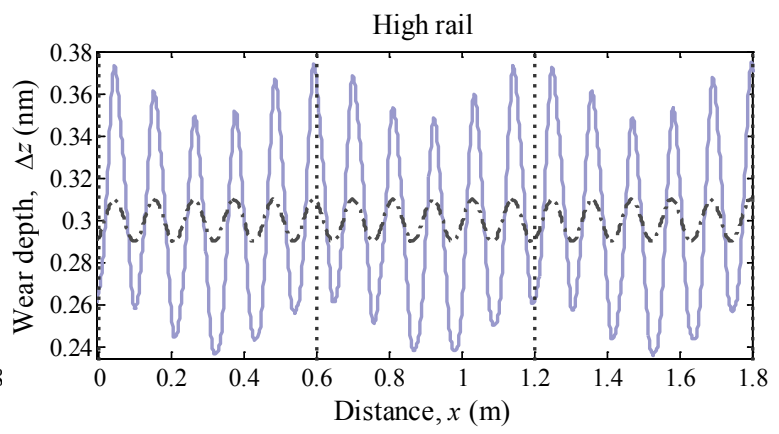

(b)

Figure 8. Wear depths on the running surfaces of both rails in a curve when the B-F modes are excited: (a) Low rail; (b) High rail. — wear depth; -.-.- rail corrugation (out of scale); '...... position of sleepers.

In Figure 9, the wear depths for both rails are represented together with the initial rail corrugation for the case in which the B-B modes are excited. Although in both rails the wear 
depth is out of phase with respect to the initial rail corrugation, it is difficult to conclude whether there would be growth of the amplitude of the initial rail corrugation or migration of the corrugation peaks as found in [15]. At the sight of the wear depths calculated, it seems that there are two main frequencies that prevail: the sleeper-passing frequency and the frequency of the initial rail corrugation. Probably, an analysis of these results in the frequency domain can be more clarifying.

In order to determine the frequency spectra of the temporal histories of the wear depth on the railheads, the Fourier transforms are computed and shown in Figure 10. To obtain these results, data corresponding to a distance of $30 \mathrm{~m}$ has been used and has been filtered with a Hanning window for minimising the spectral leakage error.

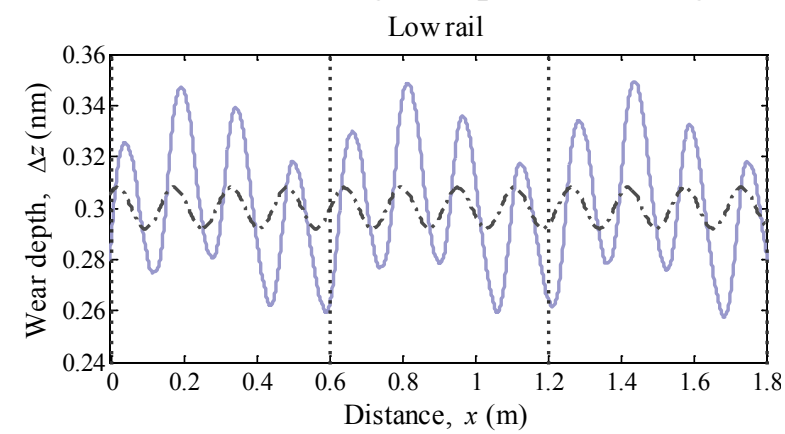

(a)

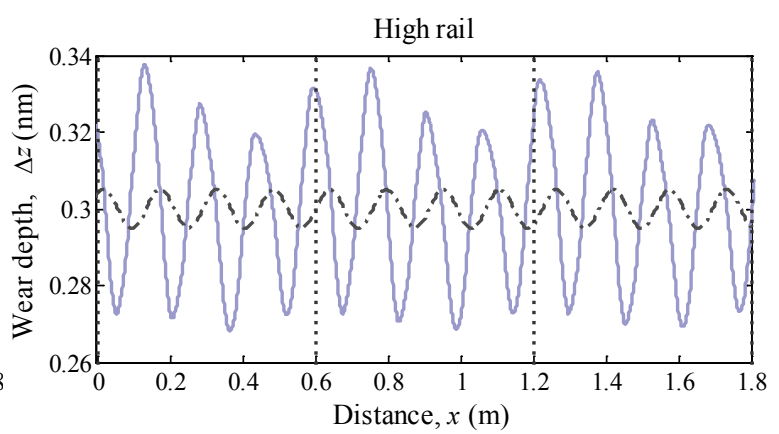

(b)

Figure 9. Wear depths on the running surfaces of both rails in a curve when the B-B modes are excited: (a) Low rail; (b) High rail. — wear depth; -.-- rail corrugation (out of scale); -..... position of sleepers.

In these spectra, it appears peaks at the sleeper-passing frequency $f_{s p}$ corresponding to the velocities under study (at $65.7 \mathrm{~Hz}$ for $V=142 \mathrm{~km} / \mathrm{h}$ in Figure 10 (a) and at $91.7 \mathrm{~Hz}$ for $V=198$ $\mathrm{km} / \mathrm{h}$ in Figure 10(b)) and its associated harmonics at $n f_{s p}$. Additionally, it can be observed another distinct peak at the frequency of the sinusoidal corrugation $f_{c}$, that is, at $360.8 \mathrm{~Hz}$ and at $352.5 \mathrm{~Hz}$ for velocities $142 \mathrm{~km} / \mathrm{h}$ and $198 \mathrm{~km} / \mathrm{h}$, respectively, and its corresponding harmonics at $n f_{c}$. Due to the nonlinearities in the vehicle-track system, there will be transfer of energy between frequencies. In the Fourier spectra in Figure 10, it can be observed that this phenomenon makes that the sleeper-passing frequency and the frequency of the sinusoidal corrugation combine resulting in new peaks of lower magnitude at frequencies $f_{c}-n f_{s p}$ and $f_{c}+n f_{s p}$.

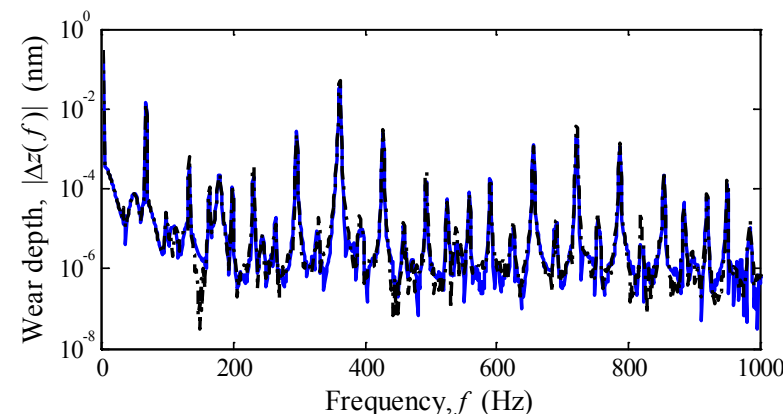

(a)

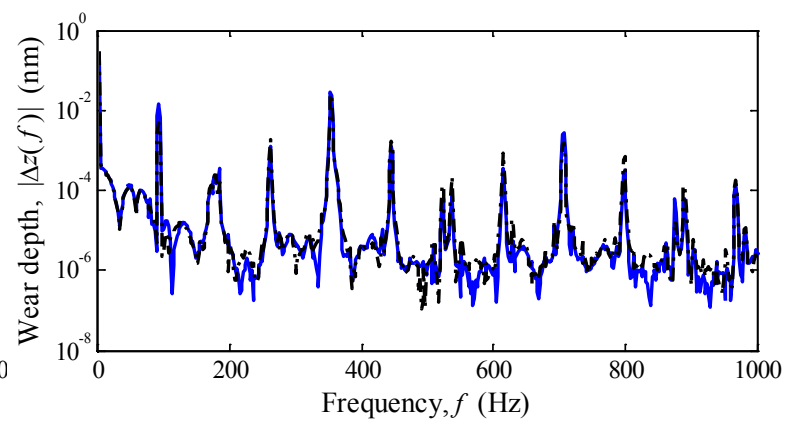

(b)

Figure 10. Fourier transform of the wear depth: (a) excitation of the B-F modes; (b) excitation of the B-B modes. - low rail; ----, high rail. 


\subsection{Broadband initial rail roughness}

In this work, global corrugation growth rates are calculated for the vehicle velocities $142 \mathrm{~km} / \mathrm{h}$ and $198 \mathrm{~km} / \mathrm{h}$ in order to identify wavelength bands in which the initial roughness may grow. The global corrugation growth rate, introduced by Hempelmann and Knothe in [3], is a non-dimensional and independent of the number of wheelset passages indicator of the growth or decrease of the amplitude of the defect at a certain wavelength or frequency. This corrugation growth rate is based on the assumption that the growth law of rail corrugation is exponential.

After computing the Fourier spectra of amplitude of the initial roughness $Z_{0}$ and the roughness after $n$ wheelset passages $Z_{n}$, these spectra have been averaged over $1 / 24$ octave bands. For the central frequency or wavelength of the $i$ th $1 / 24$ octave band, the global corrugation growth rate has been obtained through

$$
\gamma_{i}=\frac{1}{n} \ln \left(\frac{Z_{i, n}}{Z_{i, 0}}\right) \text {, with } i=1, \ldots, M .
$$

When the global corrugation growth rate is positive in a certain $1 / 24$ octave band, it means that the amplitude of the final roughness is higher than the initial roughness amplitude, which implies that the roughness would grow at the corresponding frequency. On the contrary, when the global corrugation growth is negative in a certain band, the amplitude of the roughness would decrease in that band, with which the initial roughness would be removed after a certain number of wheelset passages.

In Figure 11, the corrugation growth rates for both rails and the two vehicle velocities studied are shown. For the vehicle velocity of $142 \mathrm{~km} / \mathrm{h}$, it can be observed in Figure 11(a) that the global corrugation growth rates for wavelengths shorter than $110 \mathrm{~mm}$ take negative values, indicating that there is no growth of rail roughness for those wavelengths. However, rail roughness with a wavelength of $110 \mathrm{~mm}$ can grow on the low rail, while no growth is predicted at this wavelength on the high rail. Roughness with wavelengths in the interval 130-500 mm may grow on both rails. When the vehicle circulates at $198 \mathrm{~km} / \mathrm{h}$, similar results are found as for the velocity $142 \mathrm{~km} / \mathrm{h}$, as can be seen in Figure 11(b). In this case, roughness growth is predicted for both rails at wavelengths ranging from $200 \mathrm{~mm}$ to $600 \mathrm{~mm}$. Moreover, on the low rail, roughness would grow at a wavelength of $156 \mathrm{~mm}$, while on the high rail no roughness growth is obtained. In Figure 12, the ratio between the amplitude of the wear depth and the amplitude of the initial rail roughness evaluated in 1/24 octave bands is presented for the two velocities under study. For both vehicle velocities, it can be observed that the shorter the roughness wavelength, the higher the ratio between the wear depth amplitude and the initial roughness amplitude. This fact does not necessarily mean that the roughness of short wavelength would grow, as can be verified in Figure 11, since it depends on the phase angles between the wear depths and the initial roughness. Some distinct peaks can be noticed at a wavelength equal to the sleeper bay $(600 \mathrm{~mm})$, at wavelengths corresponding to the frequency of the B-F mode in Figure 12(a) $(110 \mathrm{~mm})$ and B-B mode in Figure 12(b) (156 mm) and at a wavelength around $35-40 \mathrm{~mm}$, which might be due to the vertical pinned-pinned antiresonance. The wide peak at about $200-250 \mathrm{~mm}$ is probably caused by the vertical antiresonance of the track in which the sleepers vibrate independently from rails and ballast, acting as dynamic vibration absorbers. 


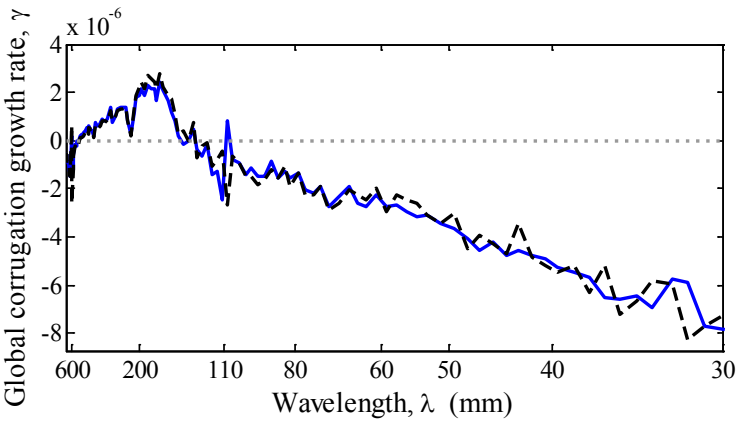

(a)

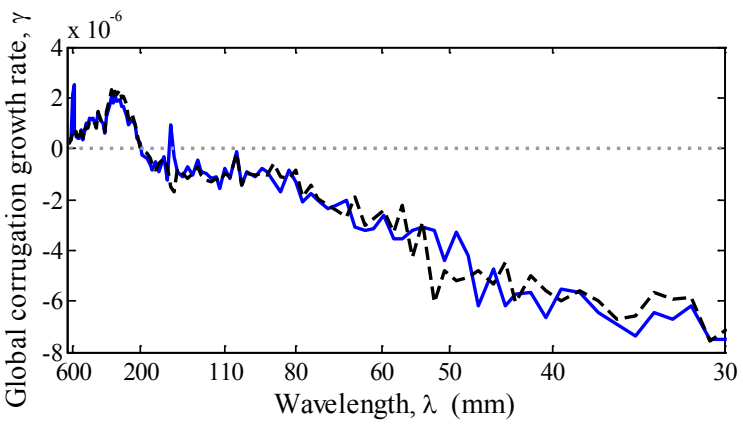

(b)

Figure 11. Global corrugation growth rate: (a) V=142 km/h; (b) V=198 km/h._—, low rail; ----; high rail.

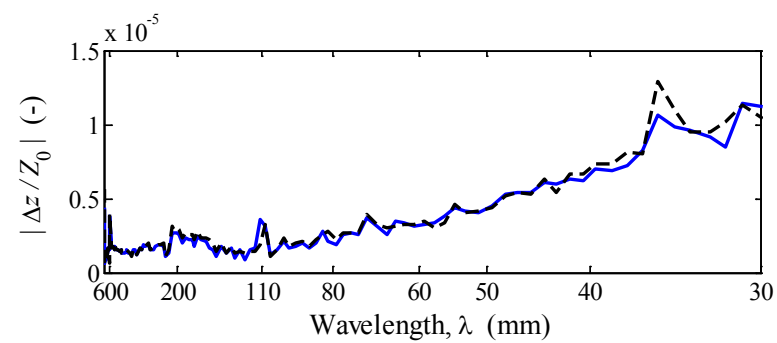

(a)

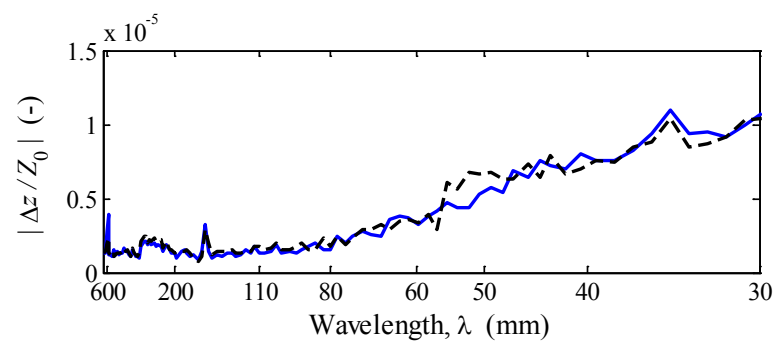

(b)

Figure 12. Wear depth amplitudes with respect to the initial roughness amplitude: (a) $V=142 \mathrm{~km} / \mathrm{h}$; (b) $V=198$ $\mathrm{km} / \mathrm{h} \mathrm{-}$, low rail; ----, high rail.

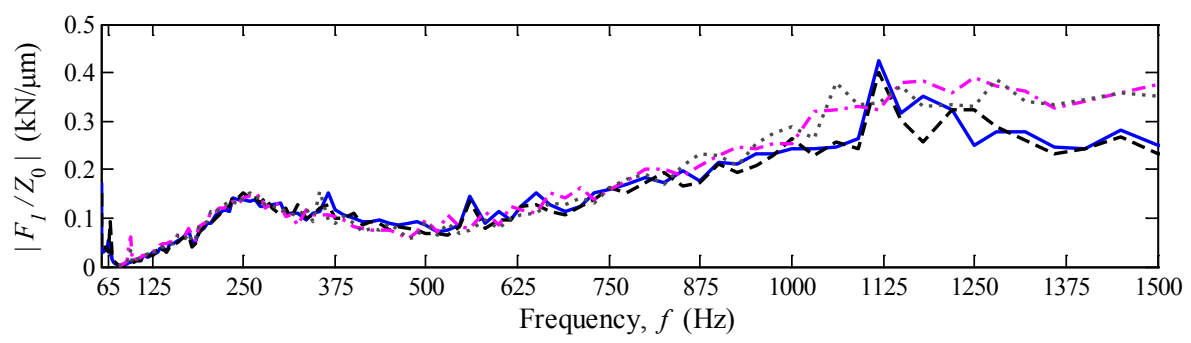

(a)

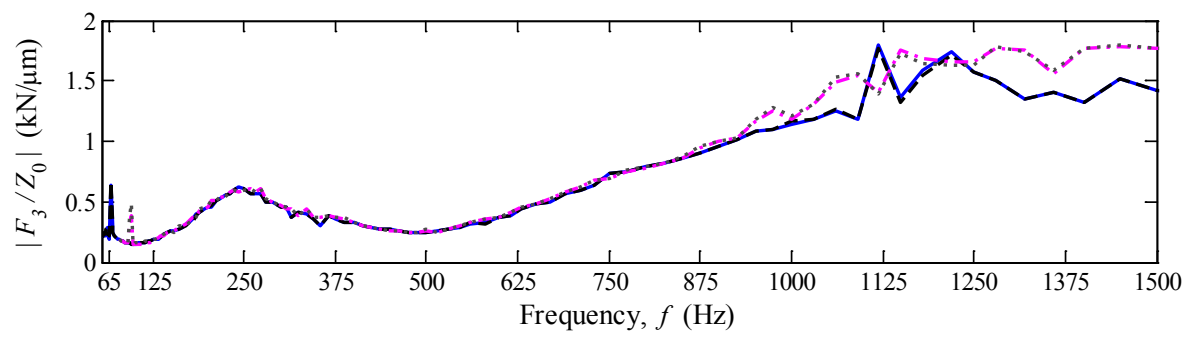

(b)

Figure 13. Amplitudes of the contact forces with respect to amplitude of initial roughness: (a) Longitudinal force;

(b) Normal force. —_, low rail at $V=142 \mathrm{~km} / \mathrm{h} ;----$, high rail at $V=142 \mathrm{~km} / \mathrm{h}$; -.-.-, low rail at $V=198 \mathrm{~km} / \mathrm{h}$; $\cdots . .$. , high rail at $V=198 \mathrm{~km} / \mathrm{h}$.

In Figure 13, the ratio between the amplitudes of contact forces and the initial roughness are plotted for both vehicle velocities in function of the excitation frequency. It can be noticed that the amplitudes of both the vertical force amplitude and the longitudinal force are higher when the excitation frequency increases. Similar results for the amplitude of the normal contact force 
with respect to the excitation frequency were obtained in [23]. The first peak appears at the sleeper-passing frequency (at $65.7 \mathrm{~Hz}$ for $V=142 \mathrm{~km} / \mathrm{h}$ and at $91.7 \mathrm{~Hz}$ for $V=198 \mathrm{~km} / \mathrm{h}$ ), the second wide peak arise around $250 \mathrm{~Hz}$ and might be produced by the antiresonance in which only the sleepers vibrate, the third and wider peak might be produced by the pinned-pinned antiresonance. In Figure 13(a), it can be seen peaks at approximately $375 \mathrm{~Hz}$, related to the excitation of the B-F and B-B modes.

\section{CONCLUSIONS}

A simulation tool has been developed to analyse the potential growth of rail corrugation under contact conditions arising when a wheelset is traversing a high radius curve. The corrugation model consists of several models connected in a feedback loop in order to account both for the short-term dynamic vehicle-track interaction, and for the long-term damage. The time-domain vehicle-track interaction model comprises a flexible rotating wheelset model, a cyclic track model based on a substructuring technique and a non-Hertzian and non-steady state three-dimensional wheel-rail contact model, based on the variational theory by Kalker. In order to improve the calculation of the distributions of contact tractions, the potential contact area is discretised into equilateral triangular elements in which the contact tractions vary linearly unlike the original method, in which piecewise constant rectangular elements are used.

The influence of the excitation of two natural frequencies of a flexible and rotating wheelset on the growth of rail corrugation and roughness has been investigated by accounting for the non-Hertzian and non-steady state effects at contact patch due to the presence of initial corrugation or roughness on the running surfaces of rails, in the frequency range from $20 \mathrm{~Hz}$ to $1500 \mathrm{~Hz}$. It has been found that when a mean positive longitudinal creepage is considered in the contact between the inner wheel and the low rail in order to simulate the contact conditions while negotiating a gentle curve, roughness growth is predicted on the low rail for the wavelengths corresponding to the B-F and B-B modes, 110 and $156 \mathrm{~mm}$, respectively. No roughness growth is obtained on the high rail under the contact conditions studied. The non-Hertzian and non-steady contact model has not predicted roughness growth for initial roughness with wavelengths shorter than $100 \mathrm{~mm}$ on both rails. These last results seem to be similar to those obtained in [13-15].

In addition, it has been shown that the vehicle-track coupled system has a nonlinear behaviour, mainly due to the wheel-rail contact. Thus, a time-domain model is needed to account for the nonlinearities of the vehicle-track system when it comes to simulate the growth of rail corrugation.

\section{ACKNOWLEDGEMENTS}

The authors acknowledge the financial contribution of the Spanish Ministry of Economy and Competitiveness through the project TRA2010-15669.

\section{REFERENCES}

[1] S. L. Grassie and J. Kalousek, Rail corrugation: characteristics, causes and treatments, Proc. Inst. Mech. Eng. F. J. Rail Rapid Transit 207 (1993), pp. 57-68.

[2] K. Hempelmann, F. Hiss, K. Knothe, B. Ripke, The formation of wear patterns on rail tread, Wear 144 (1991), pp. 179-195. 
[3] K. Hempelmann and K. Knothe, An extended linear model for the prediction of short pitch corrugation, Wear 191 (1996), pp. 161-169.

[4] S. L. Grassie and J. A. Elkins, Rail corrugation on North American transit systems, Veh. Syst. Dyn. 28 (1998), pp. 5-17.

[5] J.I. Egaña, J. Viñolas, M. Seco, Investigation of the influence of rail pad stiffness on rail corrugation on a transit system, Wear 261 (2006), pp. 216-224.

[6] A. Igeland, Railhead corrugation growth explained by dynamic interaction between track and bogie wheelsets, Proc. Inst. Mech. Eng. F. J. Rail Rapid Transit 210 (1996), pp. 11-20.

[7] I. Gómez, E. G. Vadillo, A linear model to explain short pitch corrugation on rails, Wear 255 (2003), pp. 1127-1142.

[8] C. Collette, P. Vanhonacker, R. Bastaits, D. Levy, Comparison between time and frequency studies of a corrugated curve of RER Paris network, Wear 265 (2008), pp. 1249-1258.

[9] W.J.T. Daniel, R.J. Horwood, P.A. Meehan, N. Wheatley, Analysis of rail corrugation in cornering, Wear 265 (2008), pp. 1183-1192.

[10] J. Fayos, L. Baeza, F.D. Denia and J.E. Tarancón, An Eulerian coordinate-based method for analysing the structural vibrations of a solid of revolution rotating about its main axis, J. Sound Vib. 306 (2007), pp. 618-635.

[11] L. Baeza and H. Ouyang, A railway track dynamics model based on modal substructuring and a cyclic boundary condition, J. Sound Vib. 330 (2011), pp. 75-86.

[12] J. J. Kalker, Three-dimensional elastic bodies in rolling contact, Kluwer Academic Publishers, Dordrecht, Boston London, 1990.

[13] G. Xie and S. D. Iwnicki, Simulation of wear on a rough rail using a time-domain wheel-track interaction model, Wear 265 (2008), pp.1572-1583.

[14] B. Croft, C. Jones and D. Thompson, The effect of rail dampers on roughness growth rates with multiple wear mechanisms, non-Hertzian contact and velocity dependent friction, Proceedings of the $8^{\text {th }}$ International Conference on Contact Mechanics and Wear of Rail/Wheel Systems (CM2009), Firenze, Italy, 2009.

[15] P. T. Torstensson, A. Pieringer and J.C.O. Nielsen, Simulation of rail roughness growth on small radius curves using a non-Hertzian and non-steady wheel-rail contact model, Proceedings of the $9^{\text {th }}$ International Conference on Contact Mechanics and Wear of Rail/Wheel Systems (CM2012), Chengdu, China, 2012.

[16] A. Igeland and H. Ilias, Rail head corrugation growth predictions based on non-linear high frequency vehicle/track interaction, Wear 213 (1997), pp. 90-97.

[17] P. Vila, J. Fayos and L. Baeza, Simulation of the evolution of rail corrugation using a rotating flexible wheelset model, Veh. Syst. Dyn. 49 (2011), pp. 1749-1789.

[18] K. Popp, H Kruse and I. Kaiser, Vehicle-track dynamics in the mid-frequency range, Veh. Syst. Dyn. 31 (1999), pp.423-464.

[19] K. L. Johnson, Contact Mechanics, Cambridge University Press, 1985.

[20] EN ISO3095:2005, Railway applications-acoustics measurements of noise emitted by railbound vehicles.

[21] M. Hiensch, J.C.O. Nielsen and E. Verheijen, Rail corrugation in The Netherlands-measurements and simulations, Wear 253 (2002), pp. 140-149.

[22] X. Jin, X. Xiao, Z. Wen, J. Guo, M. Zhu, An investigation into the effect of train curving on wear and contact stresses of wheel and rail. Tribol Int. 42 (2009), pp. 475-490.

[23] H. Ilias and S. Müller, A discrete-continuous track-model for wheelsets rolling over short wavelength sinusoidal rail irregularities, Veh. Syst. Dyn. 23 (1994), pp. 221-233. 